\title{
Psoriasis in childhood: effective strategies to improve treatment adherence
}

\author{
This article was published in the following Dove Press journal: \\ Psoriasis: Targets and Therapy \\ 16 March 2015 \\ Number of times this article has been viewed
}

\author{
Kara N Shah' \\ Sandra Cortina ${ }^{2,3}$ \\ Michelle M Ernst ${ }^{2}$ \\ Jessica C Kichler ${ }^{2}$ \\ 'Division of Pediatric Dermatology, \\ ${ }^{2}$ Division of Behavioral Medicine \\ and Clinical Psychology, ${ }^{3}$ Center for \\ Adherence and Self-Management, \\ Cincinnati Children's Hospital Medical \\ Center, Cincinnati, OH, USA
}

Correspondence: Kara N Shah Division of Pediatric Dermatology, Cincinnati Children's Hospital Medical Center, 3333 Burnet Avenue, MLC 3004, Cincinnati, OH 45229, USA

$\mathrm{Tel}+\mathrm{I} 5 \mathrm{I} 38037835$

Fax + I 5136365867

Email kara.shah@cchmc.org

\begin{abstract}
Psoriasis is a relatively common chronic inflammatory skin disease in children for which there is no cure. Most children have mild disease that can be managed with topical therapy as opposed to phototherapy or systemic therapy. Despite the mild presentation of psoriasis in most children, the disease can have a significant impact on quality of life due to the need for ongoing treatment, the frequently visible nature of the cutaneous manifestations, and the social stigma that is associated with psoriasis. Adherence to treatment, in particular topical therapy, is often poor in adults and compromises response to therapy and medical provider management strategies. Multiple factors that may contribute to nonadherence in adults with psoriasis have been identified, including lack of education on the disease and expectations for management, issues related to ease of use and acceptability of topical medications, and anxiety regarding possible medication side effects. There is currently no published data on adherence in the pediatric psoriasis population; however, poor adherence is often suspected when patients fail to respond to appropriate therapy. General strategies used to assess adherence in other pediatric disease populations can be applied to children with psoriasis, and interventions that reflect experience in other chronic dermatologic disorders such as atopic dermatitis may also be helpful for medical providers caring for children with psoriasis.
\end{abstract}

Keywords: adherence, psoriasis, children

\section{Introduction}

\section{Medical aspects of psoriasis}

Psoriasis is a common, chronic inflammatory dermatosis that often develops during childhood or adolescence. ${ }^{1}$ The overall prevalence in the pediatric population is about $1 \%$. Although there are effective treatments for psoriasis, there is no cure, and treatments must often be continued indefinitely in order to maintain clinical improvement. Severity of the disease may range from mild, localized disease amenable to treatment with emollients and topical medications to severe, widespread involvement that may require treatment with a complicated regimen of topical medications and/or systemic treatments such as methotrexate, tumor necrosis factor antagonists, and phototherapy. Although topical corticosteroids are the most commonly prescribed topical medication used for the treatment of psoriasis, other topical medications that may be used include topical calcineurin inhibitors, vitamin D analogs, tar, and salicylic acid. ${ }^{2}$ Given the chronicity of this disease, the often-visible skin manifestations, and the stigma attached to the diagnosis, quality of life may be significantly impacted by the disease and does not necessarily correlate with disease severity. ${ }^{3,4}$ Most patients with mild disease are managed by their primary care provider; however, those with more severe disease or 
who are not responding to treatment are often referred to a dermatologist for specialty consultation and management. When response to treatment is suboptimal, a lack of adherence to the treatment regimen must be considered.

\section{Definition of adherences and general correlates}

Although the terms adherence and compliance are often used interchangeably, over time, the use of the term adherence has become preferred. The term compliance is felt to connote a passive action on the part of the patient to follow the recommendations as dictated by the provider as opposed to a more active, interactive and dynamic process; adherence is defined as "the extent to which a person's behavior coincides with medical or health advice" and nonadherence as "late or non-initiation of the prescribed treatment, sub-optimal implementation of the dosing regimen or early discontinuation of the treatment." ${ }^{, 56}$ Formal studies of adherence in the treatment of pediatric psoriasis are lacking. Anecdotally, many parents admit and many providers suspect that adherence to the medical regimen is low, in particular for patients who fail to respond to a seemingly appropriate therapeutic regimen.

Nonadherence may be intentional, with the patient or caregiver deliberately not using treatments as prescribed due to perceived lack of efficacy, side effects, or other reasons, or unintentional, such as may result when the patient or caregivers aspire to use the treatment as prescribed, but do not understand the directions for use or forget. ${ }^{7}$ In addition to the negative impact on disease severity, nonadherence may result in increased health care expenditures, adverse effects on physical functioning and mental health, and increased school and work absenteeism and poor school performance. Both patients and providers may experience frustration, and the patient-provider relationship may suffer.

In the general pediatric population, factors that may influence adherence include the following: family demographics and functioning; parent and child characteristics; health care system and provider variables; and child health outcomes. ${ }^{8}$ Specific factors that may contribute to poor adherence in children with skin disease include the cost of medications, complicated/confusing skin care regimens, poor tolerability/ acceptability of topical medications, concern over the safety of medications, in particular topical corticosteroids, and the time required to apply medications and perform the skin care regimen. ${ }^{8}$

In order to improve patient outcomes and satisfaction with treatment regimens for the pediatric psoriasis population, decrease health care expenditures, and improve the patientprovider relationship, it is important for medical providers to understand the factors and behaviors that may contribute to poor adherence, to provide an appropriate assessment for adherence issues, and to intervene effectively. A summary of selected barriers to adherence in children with psoriasis is presented in Table 1 .

\section{Treatment adherence in children with skin disease}

Despite the negative implications of nonadherence and the presumed relatively high prevalence of nonadherence in children with dermatologic diseases, in particular with regards to topical therapy, there are very few published studies evaluating treatment adherence in this population, and there are none that specifically address adherence in pediatric psoriasis. As the majority of children with psoriasis who require treatment will be prescribed topical therapy, it may be possible to draw comparisons from data gathered from studies of children with other dermatologic disorders for whom topical medications are commonly prescribed, such as acne

Table I Barriers to treatment adherence in pediatric psoriasis

Patient factors

Co-morbid physical and/or mental health disorders

Disease-related social stigma

Poor coping skills in the context of chronic medical condition

Age-related and developmental stage-related limitations

Difficulty with transfer of treatment responsibility during adolescence

Caregiver/parent and family factors

Co-morbid physical and/or mental health disorders

Multiple caregivers/lack of primary caregiver

Family stress/chaotic home environment

Fear of medication side effects/safety (eg, corticosteroid phobia)

Negative family communication style and lack of problem-solving skills

Provider factors

Lack of provision of clear verbal and written treatment instructions

Failure to educate adequately on disease and expectations for management

Treatment factors

Poor tolerability of treatment (eg, topical medications too messy/ greasy)

Treatment too time-consuming/not convenient/negative impact on lifestyle

Complicated treatment regimen

System factors

Financial and health care insurance instability

Lack of access to alternative treatments/new technologies (eg, sprays, telehealth)

Inadequate access to mental health providers and integrated care with behavioral health

Insufficient advocacy and lack of awareness of disease in other settings (eg, schools)

Noncollaborative provider-patient/family relationship 
vulgaris and atopic dermatitis. For example, a survey-based study of 101 patients aged 1 month to 21 years with acne, atopic dermatitis, or psoriasis identified the cost of medications and concerns about side effects as common parental concerns that may limit adherence, although no assessment of adherence was included in the study design. ${ }^{9}$

Adherence to topical therapy in some dermatologic studies has been assessed through the use of a Medication Event Monitoring System (MEMS) cap, which uses a microprocessor to record the date and time that the medication container is opened (AARDEX, Zug, Switzerland). For example, in a study of 11 adolescents with acne who were instructed to apply benzoyl peroxide gel twice daily for 6 weeks, adherence decreased from $82 \%$ to $45 \%$ over the course of the study. ${ }^{10}$ Similarly, in a small study of six children with atopic dermatitis who were prescribed clocortolone pivolate $0.1 \%$ cream twice daily, self-reported adherence ranged from $71 \%$ to $97 \%$, while actual adherence as determined by use of the MEMS cap was 18\%-109\%; adherence diminished over the course of the study. ${ }^{11}$ Finally, in a prospective study of 26 children with atopic dermatitis instructed to use triamcinolone $0.1 \%$ ointment twice daily for 8 weeks, only $30 \%$ of patients were adherent with the treatment regimen as determined by use of the MEMS cap system. Of note, adherence increased around the time of office visits. ${ }^{12}$

\section{Treatment adherence in adults with psoriasis}

Given the lack of research on adherence in pediatric psoriasis, a review of the adherence literature as pertains to adult psoriasis may offer important insights. A recent review summarizing the published literature on medication adherence in adults with psoriasis found 26 studies, the majority of which reflected relatively short-term treatment and follow-up periods and over half of which $(15 / 26)$ used a self-report scale to determine patient adherence. ${ }^{13}$ There were no studies identified that evaluated adherence to the lifestyle modifications that are often recommended in psoriasis treatment, such as weight loss, limiting alcohol consumption, or smoking cessation, and none that attempted to distinguish between intentional and unintentional nonadherence. The investigators also commented on the variable and poor methodological design of most studies, which limited their interpretation of results. Despite these limitations, most of the studies still reported low adherence rates, with those studies using a dichotomous classification of adherence (14/26) noting adherence rates of only $21.6 \%-75 \% .{ }^{14-27}$ The investigators could find no consistent association between adherence and sociodemographic factors, disease severity, treatment modality (eg, topical medications, oral or injectable medications, or phototherapy), or quality of life. Four studies found a positive association between treatment adherence and clinical improvement in psoriasis symptoms (three of these studies used the same study population). ${ }^{17,27-31}$

Several studies have specifically examined the differences between subjective (self-reported) adherence and objectively measured adherence, such as by the use of the MEMS cap system or by counting or weighing unused medication, in adults with psoriasis. In two studies, subjectively measured adherence was higher than objectively measured adherence, with a subjective adherence rate of $92 \%$ and objective adherence rates ranging from $60.6 \%$ to $67 \% .^{32,33}$ In another study of 29 patients instructed to use topical 6\% salicylic acid gel twice daily, however, a mean adherence rate of $55 \%$ was seen for both the objective adherence data as determined through use of MEMS caps and unused medication weights and the subjectively reported adherence ratings. ${ }^{30}$ In an 8-week study of adults with psoriasis prescribed twice daily salicylic acid gel and tacrolimus $0.1 \%$ ointment, adherence as measured objectively through the MEMS cap demonstrated a decline from $85 \%$ to $51 \%$ over the course of the study. ${ }^{29}$ In addition, the researchers also measured adherence subjectively through patient medication logs and found that adherence was overstated in patient medication logs as compared to data obtained from use of the MEMS cap. The researchers found that for every $10 \%$ decrease in adherence, there was a 1-point increase in psoriasis disease severity as measured on a 9-point sum score incorporating erythema, scale, and plaque thickness, thereby supporting the premise that better adherence translates into clinical improvement. ${ }^{28}$

Several studies in adults with psoriasis have focused on subjective adherence ratings to understand the factors behind poor adherence from the patient perspective. In a large, online survey of 1,884 adults with psoriasis who were currently using prescription medications for the management of their psoriasis (92\% were using topical medications, $23 \%$ were using oral medications, $10 \%$ were using phototherapy, and $7 \%$ were using an injectable medication), self-reported rates of nonadherence were as follows: $46 \%$ of patients using topical medications, $25 \%$ of patients using oral medications, $35 \%$ of patients using phototherapy, and $18 \%$ of patients using an injectable medication. ${ }^{34}$ Forty-seven percent of patients reported using their medication only when they thought it was necessary. The most commonly reported reasons for poor adherence were lack of efficacy/ frustration, forgetting, being too busy/treatment regimen too 
time-consuming or inconvenient, and the unpleasant nature of topical medications (eg, too greasy or messy). Similarly, in a survey of 167 adult patients with psoriasis who were currently using a prescription topical medication for the treatment of their psoriasis, only $48 \%$ reported adherence with their prescribed medical regimen. ${ }^{35}$ The most commonly reported reasons for nonadherence were forgetting as well as using the medication only when the patient thought it was necessary. Other reasons for nonadherence included the expense of medications, lack of convenience, medications too messy/oily/sticky, too much time required to use medications, and perceived lack of efficacy. Interestingly, in a prospective study of 201 adults with psoriasis in an outpatient setting, those with facial involvement and those with more extensive psoriasis symptoms as well as those patients with more frequent (ie, complex) daily dosing schedules had lower reported adherence. ${ }^{33}$

The use of an injectable biological treatment for adults with psoriasis, such as a tumor necrosis factor antagonist, is associated with increased adherence. Although these medications are generally administered no more than twice weekly (ie, less complex dosing schedule) and are more appealing to many patients than topical medications, it is thought that increased efficacy of the medication may be the predominant explanation for the higher adherence rates, rather than improved quality of life. ${ }^{18,36}$ Given that these medications are delivered via injection or infusion, which is associated with some mild physical discomfort for the adult patient, it is not clear whether this association is also true for children and adolescents, who generally are more averse to painful interventions. In an anonymous survey of 120 adults with psoriasis using a variety of topical and systemic therapies, those with self-reported severe psoriasis were less adherent with treatment and reported greater impairment on the Dermatology Quality of Life Index as a result of their disease. ${ }^{23}$ Thirty-nine percent stated that they were "sometimes" or "never" compliant with treatment. Those patients with poor adherence were younger, more likely to have an earlier age of onset of disease, and/or to have a higher self-rated disease severity.

\section{Intervention strategies in adults with psoriasis}

As surmised from the adult psoriasis literature, patients who are more adherent with the treatment regimen achieve better clinical responses. Patients, caregivers/partners, and medical providers all need to take an active role in promoting treatment adherence. A number of different strategies have been suggested to improve treatment adherence in adults with psoriasis. One strategy focuses on building an effective patient-provider relationship, encouraging optimism with the treatment regimen, and tailoring the treatment to minimize side effects and improve acceptability by making the regimen as easy to use as possible. ${ }^{37}$ Such a strategy is also a useful framework for addressing adherence in children with psoriasis as well. Regardless of whether the patient is an adult or a child, the caregiver/partner as well as the patient must be successfully engaged. Effective communication with regards to patient education on the expectations for management and on any specific concerns regarding treatment safety and potential side effects is critical. Choosing treatments that reflect patient and caregiver/partner preferences is also important; with regards to topical medications, this means choosing medications that need only be applied once daily, if possible, and that have acceptable cosmetic and galenic properties (ie, feel pleasant on the skin and are not sticky, greasy, or malodorous). Finally, it is important that patients and their caregivers/partners develop appropriate self-management skills to manage their chronic illness consistently and independently.

There is little in the literature, however, documenting efforts to address educational or behavioral interventions to improve treatment adherence in psoriasis in adults. In an evaluation of 22 adults with psoriasis who participated in a number of small focus groups, patients expressed an interest in the provision of an individualized education plan by their health care provider, including both direct communication with a knowledgeable provider and audiovisual or written materials. ${ }^{38}$ The study investigators also inferred that based on the responses of the study participants, nonadherence was at least partially influenced by a lack of understanding of how the prescription medications worked and how to use treatments correctly, suggesting a possible focus for educational interventions.

In an Internet-based survey of adults with psoriasis who registered to watch a webcast sponsored by the National Psoriasis Foundation on the topical treatment of psoriasis, the following patient concerns were identified via online patient-submitted questions: concern for safety of topical corticosteroids, including long-term use and side effects $(30 \%$ of questions); proper use of topical medications, including how to determine the correct amount to apply (16\% of questions), the appropriate frequency of application, and when to substitute other therapies; and efficacy of different treatments (11\% of questions). ${ }^{39}$ After watching the webcast, survey participants reported the following educational information most useful: information on specific medications (24\%), 
information on safety of topical medications (18\%), and information on the range of therapeutic options (18\%). Corticosteroid phobia is also seen in other dermatologic diseases such as atopic dermatitis, where it has been associated with poor adherence, confusion regarding treatment recommendations and expectations, and lack of education on the safety of this class of medications. ${ }^{40-42}$

There is little data on the use of specific educational and/or interventional methods to improve adherence in psoriasis in adults. However, in a 12-week interventional study involving 20 adults with psoriasis that evaluated the effectiveness of daily text messaging with medication reminders and educational information determined that the patients who received the text messages also reported increased adherence (3.86 days per week at baseline and 6.46 at the end of the study versus no change in the placebo group). ${ }^{43}$ In addition, those patients who received text messaging also noted improved quality of life and clinical improvement in their psoriasis symptoms. Therefore, the limited research available on interventional strategies in adults with psoriasis suggests that simple interventions addressing adherence can positively impact functioning and clinical status of the disease.

\section{Adherence and self-management in pediatric chronic health conditions}

Across pediatric chronic health conditions, upwards of 50\% of children and adolescents are considered to be nonadherent to medical treatment regimens. ${ }^{44}$ As treatment regimens increase in complexity, the likelihood of poor adherence and health-related quality of life also rises. ${ }^{45}$ As such, improving self-management and adherence are tantamount to not only improving health outcomes, but overall pediatric adjustment to chronic disease.

When considering pediatric care, it is important to take into account the multiple relationships that occur between the psychosocial systems in which children and adolescents live. In general, children, adolescents, and even young adults are living with parents or caregivers, attending school or work, and interacting with peers as well as receiving treatment within the medical system. A broad-based model describing the dynamic process in which self-management takes place has been established. ${ }^{5}$ Within the model, the authors propose that patient-based factors (eg, developmental level and psychological adjustment) interact with family and social factors (eg, sharing of treatment responsibilities or ability to acquire adequate coverage for prescription medication) to influence adherence behavior.
Developmental factors can strongly influence adherence to medical treatment for pediatric chronic health conditions in terms of the role that the child takes in self-management. For example, adherence to a medication regimen for the young child often involves strong behavioral management skills on the part of the parent, whereas adherence for the older child or adolescent becomes a process of shared responsibility. Research indicates that adolescence is a critical period for many children with a chronic medical condition, in that adolescents often fare worse with adherence to treatment regimens than younger children across multiple pediatric conditions. ${ }^{46-48}$ With adolescence also comes increasing general responsibility, and many parents (and medical providers) begin to transition treatment responsibility to the adolescent. Whereas younger children may be safeguarded somewhat from negative health outcomes if their caregivers/parents assume active roles in treatment adherence, adolescents often assume a larger, more independent role in their selfmanagement and health care process. ${ }^{44}$ In adolescence, an increase in parent-child conflict over treatment management and adherence has also been noted. ${ }^{49}$ Parental support and continued involvement in treatment management is recommended in the transition of treatment management during adolescence.

There are also both patient and family characteristics that impact adherence in the management of pediatric chronic health conditions. An example of a patient-level factor is psychological adjustment, including the presence of mood disorders. Mood disorders, such as depression, may interfere with the ability of a pediatric patient to adhere to medical treatment regimens given the presence of symptoms such as poor concentration, fatigue, loss of interest in activities, sleep/ appetite disruption, and irritable mood..$^{50}$ As such, behavioral activation (activating oneself to participate in tasks and activities) is critical to self-management and adherence and, in turn, is a factor often impeded by depression. Similarly, parental/caregiver depression can also have negative effects on pediatric treatment management by impeding parental engagement in the demands of the medical treatment regimen. ${ }^{51}$

At the family systems level, when medical treatment is shared by family members, an understanding of treatment responsibilities becomes paramount to success in managing pediatric chronic health conditions; however, when treatment allocation is not understood or discussed collaboratively within the family, treatment adherence and management suffers. Within the family system, shared responsibility (and understanding) for self-management of medical treatment is associated with improved adherence. ${ }^{52}$ 
While interventions can be implemented for child and/or parent factors independently, intervening with both parents and children/adolescents together can be attempted, though it is more complex. Behavioral family systems therapy and multisystemic therapy have both been used with limited success in addressing family self-management and adherence to pediatric treatment. . $^{53,54}$

At a broader systems level within the medical setting, a self-management training and clinical process model has been developed at our institution to improve the way medical providers address family self-management and adherence needs in point-of-care settings. Recent state and national health care funding initiatives have prioritized health education and pediatric health behavior change in order to better meet families' self-management and adherence needs. ${ }^{55,56}$ In this way, each system works together (ie, child/adolescent, caregiver/provider, and medical provider) to influence selfmanagement, adherence, and health outcomes. ${ }^{5}$

\section{Measurement of adherence}

With current health care reform initiatives and the emphasis on clinical efficiency as well as effectiveness, the topic of valid and applicable adherence measurements in clinical care is critical for pediatric populations. ${ }^{57,58} \mathrm{~A}$ variety of assessment methods are available for use in this population, ranging from self-report to electronic monitoring (EM), the latter of which is considered the gold standard in adherence measurement. ${ }^{59,60}$ Advances in EM, such as incorporation into blister packs and tubes, are reportedly being developed but are not available for general use (Bernard Vrijens, AARDEX, personal communication, March, 2013). When such technology does become widely available, it will provide an opportunity for use of a more standardized process for the objective assessment of adherence with regards to dermatologic conditions, including psoriasis, and also with regards to possible interventions in clinical practice.

Herzer et al provided a discussion of the pros and cons of the clinical application of EM with regards to adherence in pediatric chronic health condition assessment and intervention. ${ }^{61}$ To date, only two studies and one case report have examined the effects of providing EM feedback in pediatric care; all three demonstrated positive, sustained results during treatment. ${ }^{62-64}$ While these results are encouraging, any EM study should be weighed against common limitations: EM technology is costly, requires some training, and involves extra clinical time, which may be a deterrent for some investigators and providers. The benefits of EM include improved objectivity in assessment of adherence over patient recall and the ability to identify trends in self-management, particularly with those EM devices that provide calendar feedback formats. ${ }^{61}$ Such objective information regarding adherence in a pediatric population, in turn, allows for shared decisionmaking about strategies for treatment management. ${ }^{63}$

\section{Adherence and self-management in pediatric dermatologic disorders}

Estimates of adherence rates for patients with chronic skin conditions are thought to be similar to the general pediatric chronic illness population, at about 50\%. ${ }^{9}$ Despite the recommendations in the general pediatric chronic health conditions adherence and self-management literature for the use of EM as an intervention to enhance treatment adherence, it is difficult to quantify the usage of specific topical medications as it is hard to verify the quality of the application (ie, volume actually used directly on the skin and correct distribution of application). Therefore, the subpopulation of children with a dermatologic disorder is underrepresented in the pediatric chronic illness adherence literature and warrants further attention.

Similar to the adult population, a number of barriers to adherence in pediatric patients with dermatologic disorders have been identified, such as inconvenience, time constraints, difficult/unclear instructions, and fear of side effects. ${ }^{9}$ All these adherence barriers are thought to be even more significant among children and adolescents with chronic skin conditions, due to the influence of age-related and developmental stage-related factors. ${ }^{65}$ Specifically, as children move into adolescence, they are beginning to want to take control of their bodies and may want more choices in the treatment options for their dermatologic disease. Despite this desire to take more responsibility and to have more independence, adolescents also may need more support from caregivers/parents and medical providers in developing time management skills to accommodate their medical treatment regimens into their daily routine, as the topical medications may be sticky or greasy, may impact their involvement in activities after application, and may be time-consuming to apply correctly. For example, family communication strategies and problem-solving skills to minimize conflict during this transfer of responsibility for the treatment regimen from parent to adolescent is important because this transition is often difficult to navigate for the patient and their caregivers in general chronic health care conditions and is believed to be similarly so for the pediatric dermatology population as well.

As mentioned previously, psoriasis in adults is associated with a number of psychosocial difficulties (eg, daily functioning and quality of life). A number of these psychosocial factors 
are considered barriers to adherence and are especially salient among a pediatric dermatology population. Appearance-related concerns are one of the dominant experiences of youth with psoriasis. ${ }^{66}$ Specifically, social marginalization and social reactions (or fear of these potential social reactions) to the cutaneous manifestations of disease are even more devastating to adolescents, given the "imaginary audience" and "personal fable" developmental phenomenon. Developmentally, adolescents typically believe that the whole world is watching them and that they are the only ones to have experienced negative situations. ${ }^{67}$ The negative experience of one's own skin that children/adolescents with dermatologic disorders encounter is also related to poor self-esteem, increased stress, development of psychopathology (eg, anxiety/phobias, depression), and suicidal behaviors and other functional impairments (eg, vocational/school avoidance). It is important to note, however, that the severity and intensity of the actual dermatologic symptoms is not always proportional to the level of psychosocial distress demonstrated by any given pediatric patient; therefore, clinical interventions need to be developed for all children and adolescents with dermatologic disorders, regardless of symptom severity. ${ }^{66}$

There are no published clinical interventions to date that provide recommendations for strategies in working with adherence in the pediatric psoriasis population; however, there are modest findings from the pediatric atopic dermatitis literature that can be applied to pediatric psoriasis. These atopic dermatitis interventions include the use of written action plans that include a clear outline of the medical regimen including the type of task (eg, bathing, application of topical medications, and dosing of oral medications), the frequency and location of the application of the medications, and how to adjust the intensity of treatments utilized when dermatologic symptoms are better/worse (ie, self-management) over time. ${ }^{68}$ There are examples of written action plans in the literature that use a stoplight color-coding system to aid in health literacy and understanding of the often-times complicated medical regimen associated with atopic dermatitis. ${ }^{68}$

In addition to the intervention recommendation for all medical providers to provide a detailed, simple written action plan to patients and families with atopic dermatitis, the medical provider also needs to intervene by directly educating patients and families. Five domains affecting treatment adherence in pediatric atopic dermatitis have been suggested, including 1) convenience of medication use, 2) impact of the disease versus the medication use on the lifestyle of the patients and caregivers, 3) caregiver education, 4) parent-physician relationship, and 5) alternative treatments. ${ }^{69}$ More specifically, medical providers need to be able to address these domains by demonstrating the correct application of topical medications, discussing the side effects of topical and systemic medications (eg, corticosteroid phobia), and providing a rationale for the benefits of the use of the prescribed medications and other interventions. If the caregivers/parents and the patient do not feel that their concerns and other barriers to the successful implementation of the medical regimen have been addressed, then the patient will likely be less successful with adherence and self-management. ${ }^{70}$ Medical providers need to ensure that the caregivers/parents and the patients understand the logistics of completing their medical regimen and that they have "buy in" that all aspects of the medical regimen recommendations are beneficial. Medical providers also need to ensure that they are familiar with all the potential treatment choices available to patients, strive to provide the most simple options, and enlist the help of new vehicles and technologies (eg, sprays) to provide optimal care for children and adolescents with a given dermatologic disease. ${ }^{65}$

An acknowledgment by the medical provider of the close association of psychosocial factors and stress with pediatric dermatologic disorders, including the important role of mental health providers in providing psychological interventions, is paramount. In discussions with patients and caregivers/ parents, both general pediatric medical providers as well as dermatology specialists have an equal responsibility to minimize the stigma of skin disease in our society by normalizing the typical psychosocial difficulties often associated with skin conditions and by emphasizing the importance of addressing the psychological needs of the child. The effects of a wide variety of psychological interventions for atopic dermatitis have been summarized and found that the most effective psychological interventions were a combination of stress management, relaxation and habit-reversal therapy. ${ }^{71}$ Recommendations also included the consistent use of wellestablished measures of disease symptoms and behavioral outcomes in future interventional studies for atopic dermatitis, such as the Severity Scoring of Atopic Dermatitis and the Eczema Area and Severity Index. ${ }^{71}$ An extension of the clinical recommendations regarding behavioral intervention in the pediatric atopic dermatitis literature to the pediatric psoriasis population appears relevant and prudent.

\section{Clinical recommendations to improve adherence in pediatric psoriasis}

Health care providers play a significant role in promoting treatment adherence. Table 2 provides a list of steps and 
Table 2 Interventions to increase adherence in pediatric psoriasis patients

\begin{tabular}{ll}
\hline $\begin{array}{l}\text { Educate family } \\
\text { Medical knowledge }\end{array}$ & $\begin{array}{l}\text { Discuss severity, contributing factors, and clinical } \\
\text { course of psoriasis }\end{array}$ \\
Treatment options & $\begin{array}{l}\text { Discuss topical therapies, phototherapy, and } \\
\text { systemic therapies } \\
\text { Discuss efficacy, safety concerns, and potential } \\
\text { side effects }\end{array}$ \\
& Establish expectations for improvement \\
& Define flare (consider use of photodocumentation)
\end{tabular}

interventions that medical providers can follow during treatment-related discussions to enhance patient adherence in pediatric psoriasis. First, providing appropriate education to patients and families on the pathophysiology of psoriasis and likely clinical course can enhance motivation for treatment as well as link treatment options directly to the cause and course. Using developmentally appropriate language is important so that children and adolescents of all ages can develop an understanding of their condition and treatment regimen. Subsequently, a careful review of treatment options should be forthcoming, including any possible safety concerns and side effects, in order to help families decide realistically which treatment(s) will be most successfully implemented.

Once a treatment regimen has been agreed upon by the medical provider, the patient, and the caregivers/parents, a thorough assessment of potential barriers to adherence can begin by a discussion that "normalizes" adherence difficulties. It should be recognized that nonadherence can occur even with the most committed families. If families are unable to verbalize potential challenges, a discussion of their prior treatment experiences with regards to adherence or health behaviors can highlight potential barriers, as can a review of very typical adherence challenges such as forgetfulness or time issues. Table 1 also outlines some possible solutions for common adherence issues seen within pediatric psoriasis that medical providers can discuss with families. A more detailed assessment and discussion of potential problem-solving interventions may be needed when treatments are recommended for specific daytime or nighttime routines. For example, the medical provider can review the typical bedtime routine and can help families to think more clearly about how to integrate recommended treatments and to identify potential obstacles during this time.

Financial hardship is a common concern that can affect treatment adherence and can often be mitigated through advocacy with regards to insurance company policies, particularly when providers can demonstrate the clinical evidence in support of the treatment that is being recommended. For patients who frequently forget to take an oral medication or to apply topical medications, adherence can be improved through the use of cell phone alarms, text messages, or more frequent follow-up care. The fact that patients often find the application of topical medication aversive (ie, greasy, sticky) is a significant barrier to adherence. Therefore, medical providers can highlight the importance of exploring a range of possible topical medications with patients, then problem-solve how and when to apply the topical medication to minimize this negative experience.

If psychological adjustment to the medical condition and the treatment regimen appears to be a major barrier to functioning and adherence, a referral to a psychologist may be recommended to strengthen coping skills. Similarly, addressing mental health issues or problematic family functioning and communication may be necessary for optimizing adherence. Referral to a psychologist or other mental health provider that utilizes evidence-based psychological care can positively impact adherence by treating phobic responses to seeing one's own skin if this limits willingness to apply topical medications, by treating depression that has been suppressing self-care activities, and by enhancing coping with medication injections or pill-swallowing to allow for successful implementation of systemic therapy. Families can also be referred for psychological care when family communication strategies limit the ability to effectively 
share care responsibilities, especially among adolescents, or when parental mental health issues (eg, depression) impede the provision of adequate emotional support or structure for management.

Once barriers are identified and a plan to address these barriers is in place, an assessment of patient (and caregiver/ parent) confidence, often using a 1-10 scale, can be helpful. For example, periodic assessment of the level of patient confidence can be used to highlight patient strengths ("Great, you are a 6 on the confidence level; what helps you to feel that confident?"). At the same time, that same rating can be used to enhance motivation for increased adherence ("So, you are at a 6 ; what would it take for you be at a 7 ? What would need to happen?"). If significant barriers are identified by the medical provider that cannot be addressed within the medical visit or the current medical regimen, options include switching treatment plans to one with fewer barriers or placing a referral to a psychologist to provide a more detailed assessment of adherence issues.

When the treatment plan has been refined and agreed upon by the medical provider, patient, and the caregiver/parent verbally, the use of a simple, direct written action plan that includes a clear outline of the medical regimen is important. The written action plan should include site-specific treatments (eg, scalp, face, body, hands/feet) as well as general skin care recommendations. In particular, families benefit from a clear definition of what constitutes a psoriasis "flare" and how to adjust specific treatments when intensification or de-escalation of treatment is needed. Use of photodocumentation demonstrating various manifestations of disease severity can be used as an educational and interventional tool with families. Given that many families may come to their first visit with untreated "flaring" psoriatic disease, this may be an optimal time to initiate patient-specific photodocumentation, with subsequent photodocumentation performed at follow-up evaluations as the disease improves. Review of photodocumentation over time allows for patients and caregivers/parents to be able to better judge response to therapeutic interventions, which can be initially underestimated.

Finally, the importance of close medical follow-up cannot be overstated, especially given the observation that treatment adherence increases around the time-of-office appointments. While frequent visits for the purpose of enhancing adherence is not sustainable in the long term, when new adherence behaviors are being developed, follow-up within 2-4 weeks can help to positively reward self-care while also offering the opportunity for early reeducation or barrier mitigation. Importantly, acknowledgment by the medical provider that there are likely to be some adherence challenges is important, and maintaining a constructive solution-focused (rather than punitive) approach is essential.

\section{Innovation in pediatric psoriasis care}

The current health care reform initiative highlights the provision of integrated psychosocial and medical care of patients. ${ }^{72}$ Many skin conditions present a considerable psychosocial challenge, and psychosocial stress can potentially worsen psoriasis. ${ }^{66} \mathrm{~A}$ coordinated treatment approach can promote successful and comprehensive care by addressing the biological, psychological, and social aspects of psoriasis, thereby facilitating optimal adherence and patient quality-oflife outcomes. "Psychodermatology clinics", during which patients have access to both dermatologic as well as mental health providers, are one innovative mechanism for providing integrated care. Advantages to this approach include decreased stigma of addressing mental health concerns, more immediate provision of (brief) psychological interventions, and increased access for families. ${ }^{73}$ A model utilizing integrated care in the treatment of children with atopic dermatitis has been described by LeBovidge et al and can be extended to pediatric psoriasis. ${ }^{74}$ In this clinic, all families were offered the opportunity to meet with a psychologist, with $70 \%$ of families utilizing this service. Of note, adherence concerns were highlighted as one of the primary reasons for patients requesting to meet with the psychologist (eg, caregiver/ parent feeling overwhelmed with managing the child's dermatologic condition and difficulty following through with treatment recommendations).$^{74}$ Challenges to this provision of integrated care include sustaining a financial business model, although the utilization of health and behavior billing codes, which allow for insurance payment for psychological care for medical diagnoses, has been successfully used in integrative care models. ${ }^{74,75}$

Telehealth is another means of providing innovative quality care and has been used effectively to foster adherence across other pediatric chronic illness populations. ${ }^{76}$ Telehealth may be particularly useful in managing conditions that require acute visual assessment of skin symptoms, such as pediatric psoriasis. Recent investigations have documented equivalent outcomes between adult psoriasis patients seen for in-person office visits versus those who submitted photodocumentation of skin status via email and who subsequently received emailed recommendations and prescriptions. ${ }^{77}$ "Teledermatology" has been considered an adherence-enhancing mechanism by facilitating 
home-monitoring, empowering patients, and strengthening patient-physician collaboration, with some evidence of a positive impact on adherence behaviors borne out in the literature. ${ }^{43}$ Both adult psoriasis patients and medical providers have reported both benefit and satisfaction with the use of mobile teledermatology. ${ }^{78}$

Another innovative intervention for pediatric psoriasis is through the further use and development of EM measurement in medical clinics. Although EM, including the use of MEMS caps, has been utilized in small studies in the adult psoriasis literature with some success, there are no examples of the use of MEMS caps in the pediatric psoriasis literature. Despite some of the limitations of the current EM methodology, Balkrishnan et al demonstrated that commonly used skin ointments, gels and creams could be placed in bottles fitted with MEMS caps without loss of stability or efficacy. ${ }^{32}$ Therefore, medical providers working with children with psoriasis may be able integrate the use of MEMS caps into their routine clinical care to identify patient-specific patterns of medication administration and to enhance adherence and treatment planning with patients and their caregivers/parents. This is especially useful within the pediatric psoriasis population as the vast majority of children and adolescents with psoriasis utilize topical medications and routine outpatient follow-up visits occur regularly.

\section{Future directions}

There are many opportunities for future research in adherence in pediatric psoriasis. Specific areas for future inquiry include the following: incorporating patient and caregiver/parent preference in the selection of specific treatments; identifying the impact of psychosocial distress (eg, body image issues) in coping/adjusting to the disease; assessing barriers to adherence and the development of effective written care plans; and further evaluation of innovative care models such as integrated psychodermatology clinics, teledermatology technology, and EM methodology. Evaluating the effectiveness of established interventions that have been utilized with other health behaviors and that promote adherence such as motivational interviewing or of newer treatment approaches such as acceptance and commitment therapy, may both strengthen treatment outcomes as well as elucidate key motivational factors relevant to self-management and adherence in pediatric psoriasis. ${ }^{79,80}$

\section{Conclusion}

Pediatric psoriasis is a chronic skin condition that may be associated with significant disease burden and negative physiologic and psychosocial outcomes. As such, adherence issues are present for children with psoriasis as they are with other chronic health conditions and may be exacerbated by the characteristics of topical medications commonly used, the visible nature of the disorder on the skin, and the impact of stress on the severity of the disease. It is prudent for medical providers to assume that barriers to adherence are present for each and every patient, and it is incumbent on providers to actively address these barriers both through verbal communication and in written action plans in order to increase patient and parents confidence and self-management skills. Fortunately, health provider-directed adherence interventions have been shown to improve health behaviors in pediatric patients with chronic health conditions, and these interventions can be extended to the pediatric psoriasis population.

\section{Disclosure}

The authors report no conflicts of interest in this work.

\section{References}

1. Tollefson MM. Diagnosis and management of psoriasis in children. Pediatr Clin North Am. 2014;61(2):261-277.

2. Shah KN. Diagnosis and treatment of pediatric psoriasis: current and future. Am J Clin Dermatol. 2013;14(3):195-213.

3. de Jager ME, van de Kerkhof PC, de Jong EM, Seyger MM. A crosssectional study using the Children's Dermatology Life Quality Index (CDLQI) in childhood psoriasis: negative effect on quality of life and moderate correlation of CDLQI with severity scores. Br J Dermatol. 2010;163(5):1099-1101.

4. Ganemo A, Wahlgren CF, Svensson A. Quality of life and clinical features in Swedish children with psoriasis. Pediatr Dermatol. 2011;28(4):375-379.

5. Modi AC, Pai AL, Hommel KA, et al. Pediatric self-management: a framework for research, practice, and policy. Pediatrics. 2012;129(2): e473-e485.

6. Vrijens B, De Geest S, Hughes DA, et al; ABC Project Team. A new taxonomy for describing and defining adherence to medications. $\mathrm{Br} J$ Clin Pharmacol. 2012;73(5):691-705.

7. Horne R. Representations of medication and treatment: advances in theory and measurement. In: Petrie KJ, Weinman JA, editors. Perceptions of Health and Illness. Amsterdam, The Netherlands: Harwood Academic Publishers; 1997:155-188.

8. Ou HT, Feldman SR, Balkrishnan R. Understanding and improving treatment adherence in pediatric patients. Semin Cutan Med Surg. 2010;29(2):137-140.

9. Ellis RM, Koch LH, McGuire E, Williams JV. Potential barriers to adherence in pediatric dermatology. Pediatr Dermatol. 2011;28(3): 242-244.

10. Yentzer BA, Alikhan A, Teuschler H, et al. An exploratory study of adherence to topical benzoyl peroxide in patients with acne vulgaris. J Am Acad Dermatol. 2009;60(5):879-880.

11. Conde JF, Kaur M, Fleischer AB Jr, Tusa MG, Camacho F, Feldman SR. Adherence to clocortolone pivalate cream $0.1 \%$ in a pediatric population with atopic dermatitis. Cutis. 2008;81(5):435-441.

12. Krejci-Manwaring J, Tusa MG, Carroll C, et al. Stealth monitoring of adherence to topical medication: adherence is very poor in children with atopic dermatitis. J Am Acad Dermatol. 2007;56(2):211-216.

13. Thorneloe RJ, Bundy C, Griffiths CE, Ashcroft DM, Cordingley L. Adherence to medication in patients with psoriasis: a systematic literature review. Br J Dermatol. 2013;168(1):20-31. 
14. Altobelli E, Marziliano C, Fargnoli MC, et al. Current psoriasis treatments in an Italian population and their association with sociodemographical and clinical features. J Eur Acad Dermatol Venereol. 2012;26(8):976-982.

15. Brown KK, Rehmus WE, Kimball AB. Determining the relative importance of patient motivations for nonadherence to topical corticosteroid therapy in psoriasis. J Am Acad Dermatol. 2006;55(4): 607-613.

16. Chan SA, Hussain F, Lawson LG, Ormerod AD. Factors affecting adherence to treatment of psoriasis: comparing biologic therapy to other modalities. J Dermatolog Treat. 2013;24(1):64-69.

17. Evers AW, Kleinpenning MM, Smits T, et al. Treatment nonadherence and long-term effects of narrowband UV-B therapy in patients with psoriasis. Arch Dermatol. 2010;146(2):198-199.

18. Fouere S, Adjadj L, Pawin H. How patients experience psoriasis: results from a European survey. J Eur Acad Dermatol Venereol. 2005; 19(Supp1 3):2-6.

19. Gokdemir G, Ari S, Koslu A. Adherence to treatment in patients with psoriasis vulgaris: Turkish experience. J Eur Acad Dermatol Venereol. 2008;22(3):330-335.

20. Kamangar F, Isip L, Bhutani T, et al. How psoriasis patients perceive, obtain, and use biologic agents: Survey from an academic medical center. J Dermatolog Treat. 2013;24(1):13-24.

21. Renzi C, Picardi A, Abeni D, et al. Association of dissatisfaction with care and psychiatric morbidity with poor treatment compliance. Arch Dermatol. 2002;138(3):337-342.

22. Ribera M, Dauden E, Puig L, et al. [Design and validation of a questionnaire to measure treatment satisfaction in patients with moderate-to-severe psoriasis: the NEODERMA study]. Actas Dermo-Sifiliograficas. 2011;102(1):28-38. Spanish.

23. Richards HL, Fortune DG, O’Sullivan TM, Main CJ, Griffiths CE. Patients with psoriasis and their compliance with medication. $J \mathrm{Am}$ Acad Dermatol. 1999;41(4):581-583.

24. van de Kerkhof PC, de Hoop D, de Korte J, Cobelens SA, Kuipers MV. Patient compliance and disease management in the treatment of psoriasis in the Netherlands. Dermatology. 2000;200(4):292-298.

25. van de Kerkhof PC, Franssen M, de La Brassine M, Kuipers M. Calcipotriol cream in the morning and ointment in the evening: a novel regimen to improve compliance. J Dermatolog Treat. 2001;12(2): 75-79.

26. van de Kerkhof PC, Steegers-Theunissen RP, Kuipers MV. Evaluation of topical drug treatment in psoriasis. Dermatology. 1998;197(1): 31-36.

27. Lynde CW, Gupta AK, Guenther L, Poulin Y, Levesque A, Bissonnette R. A randomized study comparing the combination of nbUVB and etanercept to etanercept monotherapy in patients with psoriasis who do not exhibit an excellent response after 12 weeks of etanercept. J Dermatolog Treat. 2012;23(4):261-267.

28. Carroll CL, Feldman SR, Camacho FT, Balkrishnan R. Better medication adherence results in greater improvement in severity of psoriasis. Br J Dermatol. 2004;151(4):895-897.

29. Carroll CL, Feldman SR, Camacho FT, Manuel JC, Balkrishnan R. Adherence to topical therapy decreases during the course of an 8-week psoriasis clinical trial: commonly used methods of measuring adherence to topical therapy overestimate actual use. J Am Acad Dermatol. 2004;51(2):212-216.

30. Feldman SR, Camacho FT, Krejci-Manwaring J, Carroll CL, Balkrishnan R. Adherence to topical therapy increases around the time of office visits. J Am Acad Dermatol. 2007;57(1):81-83.

31. Lecha M, Mirada A, Lopez S, Artes M, TOP. Research Group. Tacalcitol in the treatment of psoriasis vulgaris: the Spanish experience. $J$ Eur Acad Dermatol Venereol. 2005;19(4):414-417.

32. Balkrishnan R, Carroll CL, Camacho FT, Feldman SR. Electronic monitoring of medication adherence in skin disease: results of a pilot study. J Am Acad Dermatol. 2003;49(4):651-654.

33. Zaghloul SS, Goodfield MJ. Objective assessment of compliance with psoriasis treatment. Arch Dermatol. 2004;140(4):408-414.
34. Bewley A, Burrage DM, Ersser SJ, Hansen M, Ward C. Identifying individual psychosocial and adherence support needs in patients with psoriasis: a multinational two-stage qualitative and quantitative study. J Eur Acad Dermatol Venereol. 2014;28(6):763-770.

35. Feldman SR. Disease burden and treatment adherence in psoriasis patients. Cutis. 2013;92(5):258-263.

36. Bhosle MJ, Feldman SR, Camacho FT, Timothy Whitmire J, Nahata MC, Balkrishnan R. Medication adherence and health care costs associated with biologics in Medicaid-enrolled patients with psoriasis. J Dermatolog Treat. 2006;17(5):294-301.

37. Richards HL, Fortune DG, Griffiths CE. Adherence to treatment in patients with psoriasis. J Eur Acad Dermatol Venereol. 2006;20(4): 370-379.

38. Ersser SJ, Cowdell FC, Latter SM, Healy E. Self-management experiences in adults with mild-moderate psoriasis: an exploratory study and implications for improved support. Br J Dermatol. 2010;163(5): 1044-1049.

39. Martin SL, McGoey ST, Bebo BF Jr, Feldman SR. Patients' educational needs about topical treatments for psoriasis. J Am Acad Dermatol. 2013;68(6):e163-e168.

40. Aubert-Wastiaux H, Moret L, Le Rhun A, et al. Topical corticosteroid phobia in atopic dermatitis: a study of its nature, origins and frequency. Br J Dermatol. 2011;165(4):808-814.

41. Kojima R, Fujiwara T, Matsuda A, et al. Factors associated with steroid phobia in caregivers of children with atopic dermatitis. Pediatr Dermatol. 2013;30(1):29-35.

42. Smith SD, Hong E, Fearns S, Blaszczynski A, Fischer G. Corticosteroid phobia and other confounders in the treatment of childhood atopic dermatitis explored using parent focus groups. Australas J Dermatol. 2010;51(3):168-174.

43. Balato N, Megna M, Di Costanzo L, Balato A, Ayala F. Educational and motivational support service: a pilot study for mobile-phone-based interventions in patients with psoriasis. Br J Dermatol. 2013;168(1): 201-205.

44. Rapoff M. Adherence to Pediatric Medical Regimens. 2nd ed. New York, NY: Springer Science + Business Media; 2010.

45. Roberts M, Steele R. Handbook of Pediatric Psychology. 4th ed. New York, NY: The Guilford Press; 2009.

46. Anderson BJ, Brackett J, Ho J, Laffel LMB. An office-based intervention to maintain parent-adolescent teamwork in diabetes management. Impact on parent involvement, family conflict, and subsequent glycemic control. Diabetes Care. 1999;22(5):713-721.

47. Feinstein S, Keich R, Becker-Cohen R, Rinat C, Schwartz SB, Frishberg Y. Is noncompliance among adolescent renal transplant recipients inevitable? Pediatrics. 2005;115(4):969-973.

48. Masterson TL, Wildman BG, Newberry BH, Omlor GJ. Impact of age and gender on adherence to infection control guidelines and medical regimens in cystic fibrosis. Pediatr Pulmonol. 2011;46(3):295-301.

49. Hilliard ME, Guilfoyle SM, Dolan LM, Hood KK. Prediction of adolescents' glycemic control 1 year after diabetes-specific family conflict: the mediating role of blood glucose monitoring adherence. Arch Pediatr Adolesc Med. 2011;165(7):624-629.

50. DiMatteo M, Lepper HS, Croghan TW. Depression is a risk factor for noncompliance with medical treatment: meta-analysis of the effects of anxiety and depression on patient adherence. Arch Intern Med. 2000;160(14):2101-2107.

51. Cameron LD, Young MJ, Wiebe DJ. Maternal trait anxiety and diabetes control in adolescents with type 1 diabetes. J Pediatr Psychol. 2007;32(7):733-744.

52. Pai AL, Gray E, Kurivial K, Ross J, Schoborg D, Goebel J. The allocation of treatment responsibility scale: a novel tool for assessing patient and caregiver management of pediatric medical treatment regimens. Pediatr Transplant. 2010;14(8):993-999.

53. Ellis D, Naar-King S, Templin T, et al. Multisystemic therapy for adolescents with poorly controlled type 1 diabetes: reduced diabetic ketoacidosis admissions and related costs over 24 months. Diabetes Care. 2008;31(9):1746-1747. 
54. Wysocki T, Harris MA, Buckloh LM, et al. Randomized trial of behavioral family systems therapy for diabetes: maintenance of effects on diabetes outcomes in adolescents. Diabetes Care. 2007;30(3):555-560.

55. Adherence Research Network. 2011. Available from: http:// obssr.od.nih.gov/scientific_areas/health_behaviour/adherence/ adherenceresearchnetwork.aspx.

56. Newacheck PW, McManus MA, Fox HB. Prevalence and impact of chronic illness among adolescents. Am J Dis Child. 1991;145(12): 1367-1373.

57. Cohen LL, La Greca AM, Blount RL, Kazak AE, Holmbeck GN, Lemanek KL. Introduction to special issue: evidence-based assessment in pediatric psychology. J Pediatr Psychol. 2008;33(9):911-915.

58. McQuaid EL. Commentary: integrating lessons from evidence-based assessment of adherence into clinical practice. J Pediatr Psychol. 2008;33(9):937-938.

59. Drotar D. Promoting Adherence to Medical Treatment in Chronic Childhood Illness. Mawhaw, NJ: Lawrence Erlbaum Associates; 2000.

60. Duncan CL, Mentrikoski JM, Wu YP, Fredericks EM. Practice-based approach to assessing and treating nonadherence in pediatric regimens. Clin Pract Pediatr Psychol. 2014;2(3):322-326.

61. Herzer M, Ramey C, Rohan J, Cortina S. Incorporating electronic monitoring feedback into clinical care: a novel and promising adherence promotion approach. Clin Child Psychol Psychiatry. 2012;17(4): 505-518.

62. Otsuki M, Eakin MN, Rand CS, et al. Adherence feedback to improve asthma outcomes among inner-city children: a randomized trial. Pediatrics. 2009;124(6):1513-1521.

63. Cortina S, Somers M, Rohan JM, Drotar D. Clinical effectiveness of comprehensive psychological intervention for nonadherence to medical treatment: a case series. J Pediatr Psychol. 2013;38(6):649-663.

64. Hilliard ME, Ramey C, Rohan JM, Drotar D, Cortina S. Electronic monitoring feedback to promote adherence in an adolescent with Fanconi anemia. Health Psychol. 2011;30(5):503-509.

65. Wheeler T. Teens with chronic skin: are we speaking their language? Br J Nurs. 2010;19(19):1214-1214.

66. Levenson JL. Psychiatric issues in dermatology, part 1: atopic dermatitis and psoriasis. Prim psychiatry. 2008;15(7):35-38.

67. Elkind D, Bowen R. Imaginary audience behavior in children and adolescents. Dev Psychol. 1979;15(1):38.
68. Rork JF, Sheehan WJ, Gaffin JM, et al. Parental response to written eczema action plans in children with eczema. Arch Dermatol. 2012;148(3):391-392.

69. Fenerty SD, O’Neill JL, Gustafson CJ, Feldman SR. Maternal adherence factors in the treatment of pediatric atopic dermatitis. JAMA Dermatol. 2013;149(2):229-231.

70. Dabade TS, Feldman SR. We must think outside the box to understand nonadherence. Pediatr Dermatol. 2011;28(3):353-353.

71. Chida Y, Steptoe A, Hirakawa N, Sudo N, Kubo C. The effects of psychological intervention on atopic dermatitis. Int Arch Allergy Immunol. 2007;144(1):1-9.

72. Tynan WD, Woods KE. Emerging issues: psychology's place in the primary care pediatric medical home. Clin Pract Pediatric Psychol. 2013;1:380-385.

73. Orion E, Feldman B, Ronni W, Orit BA. A psychodermatology clinic: the concept, the format, and our observations from Israel. Am J Clin Dermatol. 2012;13(2):97-101.

74. LeBovidge JS, Kelley SD, Lauretti A, et al. Integrating medical and psychological health care for children with atopic dermatitis. J Pediatr Psychol. 2007;32(5):617-625.

75. Claar RL, Kaczynski KJ, Lyons MM, Lebel AA. Commentary: health and behavior codes in a pediatric headache program: reimbursement data and recommendations for practice. J Pediatr Psychol. 2012;37(5): 509-513.

76. Wu YP, Hommel KA. Using technology to assess and promote adherence to medical regimens in pediatric chronic illness. $J$ Pediatr. 2014;164(4):922-927.

77. Chambers CJ, Parsi KK, Schupp C, Armstrong AW. Patient-centered online management of psoriasis: a randomized controlled equivalency trial. J Am Acad Dermatol. 2012;66(6):948-953.

78. Frühauf J, Schwantzer G, Ambros-Rudolph CM, et al. Pilot study on the acceptance of mobile teledermatology for the home monitoring of high-need patients with psoriasis. Australas J Dermatol. 2012;53(1): $41-46$.

79. Gregg JA, Callaghan GM, Hayes SC, Glenn-Lawson JL. Improving diabetes self-management through acceptance, mindfulness, and values: a randomized controlled trial. J Consult Clin Psychol. 2007;75(2):336

80. Martins RK, McNeil DW. Review of motivational interviewing in promoting health behaviors. Clin Psychol Rev. 2009;29(4):283-293.
Psoriasis: Targets and Therapy

\section{Publish your work in this journal}

Psoriasis: Targets and Therapy is international, peer-reviewed, open access journal focusing on psoriasis, nail psoriasis, psoriatic arthritis and related conditions, identification of therapeutic targets and the optimal use of integrated treatment interventions to achieve improved outcomes and quality of life. The manuscript management system

\section{Dovepress}

is completely online and includes a very quick and fair peer-review system. Visit http://www.dovepress.com/testimonials.php to read real quotes from published authors. 